\title{
Frequency division multiplexing for very high capacity transmission in bandwidth-limited systems
}

\author{
Alberto Gatto, Paola Parolari, and Pierpaolo Boffi \\ POLITECNICO DI MILANO, Dip. Elettronica, Informazione e Bioingegneria, PoliCom Lab, via Ponzio 34/5 - 20133 Milan (ITALY) \\ pierpaolo.boffi@polimi.it
}

\begin{abstract}
FDM is shown to achieve high capacity facing system bandwidth limitations and exploiting its flexibility to allocate the subcarriers, also with an effective combination with MDM, in different applications, including PONs and mobile fronthaul.

OCIS codes: (060.2330) Fiber optics communications; (060.4080) Modulation.
\end{abstract}

\section{Introduction}

The traffic demand for very high capacity transmission is rapidly increasing in short reach optical links, e.g. datacenter interconnections, and in medium reach optical links, e.g. access networks and mobile fronthaul. For this kind of applications the employment of cost-effective, energy-efficient and reduced-complexity solutions is mandatory and the use of the standard approach based on single optical carrier modulation, widely used for longhaul transmission, does not seem to be the best choice. The exploitation of multicarrier approach combined with direct modulation and detection could be advantageous, supported by the availability of high-speed digital signal processing (DSP), enabling a suitable signal spectrum design. Orthogonal frequency division multiplexing (OFDM) and discrete multitone (DMT) techniques lead to a high spectral efficiency thanks to the partially subcarrier overlapping and bit-loading operation. However, they show the disadvantage of high peak-to-average power ratio (PAPR), that in case of non-linear response of the employed system components and nonlinear fiber impairments can induce significant signal distortions, limiting the system performance [1]. On the other hand, frequency division multiplexing (FDM) appears as an interesting alternative thanks to its intrinsic flexibility. By exploiting FDM modulation, the spectrum portion and shape, the modulation format and the baud rate of each subcarrier can be adapted to the system characteristics, including the non-linear and bandwidth-limited behavior of the employed components, the channel crosstalk or the application requirements.

In this work we show the FDM appeal for both point-to-point high capacity interconnections and for the nextgeneration access point-to-multipoint applications. In particular, we will demonstrate that by tailoring the modulation format, order and power of the various FDM subcarriers it is possible to overcome the bandwidth limitation and the not-uniform frequency response of a directly detected uncompensated system, as by employing DMT modulation supported by bit-loading. Dynamic and flexible subcarrier assignment according to the subscriber type and to the requested services is obtained in the upstream (US) transmission in a passive optical network (PON), where multiple access is based on FDM. Moreover, the adaptation of the FDM subcarrier allocation and modulation will be exploited together with effective multiplexing techniques, such as mode division multiplexing (MDM), to support the mobile fiber fronthaul of future $5 \mathrm{G}$ mobile networks with a huge number of antenna elements.

\section{High capacity FDM transmission in bandwidth-limited VCSEL-based data interconnections}

Future data network scenario for reach up to few tens of kms will be very sensitive to cost, footprint and power consumption, requiring the employment of cost-effective and energy-efficient devices. Long-wavelength verticalcavity surface-emitting lasers (VCSELs) are good candidates as optical sources, but to overcome their E/O bandwidth limitation multicarrier modulation has to be adopted in order to achieve a high spectral efficiency. Besides, FDM subcarrier allocation and modulation can be matched to the system non-uniform frequency response induced by direct modulation and detection and by the propagation over an uncompensated standard single-mode fiber (SSMF). As a proof of principle, in our experimentation we take into account a low-cost 1580-nm single-mode VCSEL [2] with 8-MHz linewidth, standard frequency chirp (linewidth enhancement factor $\alpha \approx 5.5$ and laserspecific adiabatic constant $\left.\kappa \approx 10^{13}\right), 0.5-\mathrm{mW}$ emitted power and about $5-\mathrm{GHz}$ electro-optical bandwidth. At the receiver side we employ direct detection by means of a $12-\mathrm{GHz}$ PIN receiver. The laser frequency chirp and the double-sideband spectrum due to VCSEL direct modulation induce a non-uniform frequency response of the propagation over uncompensated SSMF with fading dips dependent on the cumulated chromatic dispersion. Thanks to FDM exploitation, we fit the allocation and modulation of the signal subcarriers to match the VCSEL band limitation and the non-uniform frequency response of the system. As a function of the SSMF reach, we fill the spectral dips by FDM subcarriers characterized by lower spectral efficiency and more robust modulation formats (BPSK or QPSK). Very high capacities (34-Gb/s data rate over $100 \mathrm{~m}, 28-\mathrm{Gb} / \mathrm{s}$ over $10 \mathrm{~km}, 25-\mathrm{Gb} / \mathrm{s}$ over $20 \mathrm{~km}$ ) 
have been experimentally achieved [3], adopting 10 FDM 0-roll-off Nyquist shaped subcarriers, each one modulated at 1 Gbaud and evenly spaced without inter-subcarrier guard-band. Also 9-taps FFE and 4-taps DFE equalization is considered at the receiver. Fig. 1 a) shows the received electrical spectrum and the BERs corresponding to the 10 subcarriers (their modulation order is shown above the graphic) in case of $20-\mathrm{km}$ SSMF propagation causing a frequency fading around $4 \mathrm{GHz}$. Thanks to the electrical equalization of the transmitted power spectra, no significant spread among the subcarriers is visible, leading to BER below the 7\%-overhead FEC threshold of $3.8 \cdot 10^{-3}$. Alternately, DMT modulation (Fig. $1 \mathrm{~b}$ ) through the use of a large number of subcarriers can optimize the transmitted capacity with the automatic bit and power loading; however, when the number of subcarriers increases, the complexity, the latency at the transmitter side and PAPR distortions considerably increase as well. As shown in Fig. 1 c), for lower SSMF reach (e.g. $100 \mathrm{~m}$ ) the supported capacity is higher for DMT than for FDM modulation, but increasing the propagation distance (as in case of $20 \mathrm{~km}$ ) FDM and 256-subcarriers DMT performance become similar (experimented DMT mean BER of $3.43 \cdot 10^{-3}$ ).

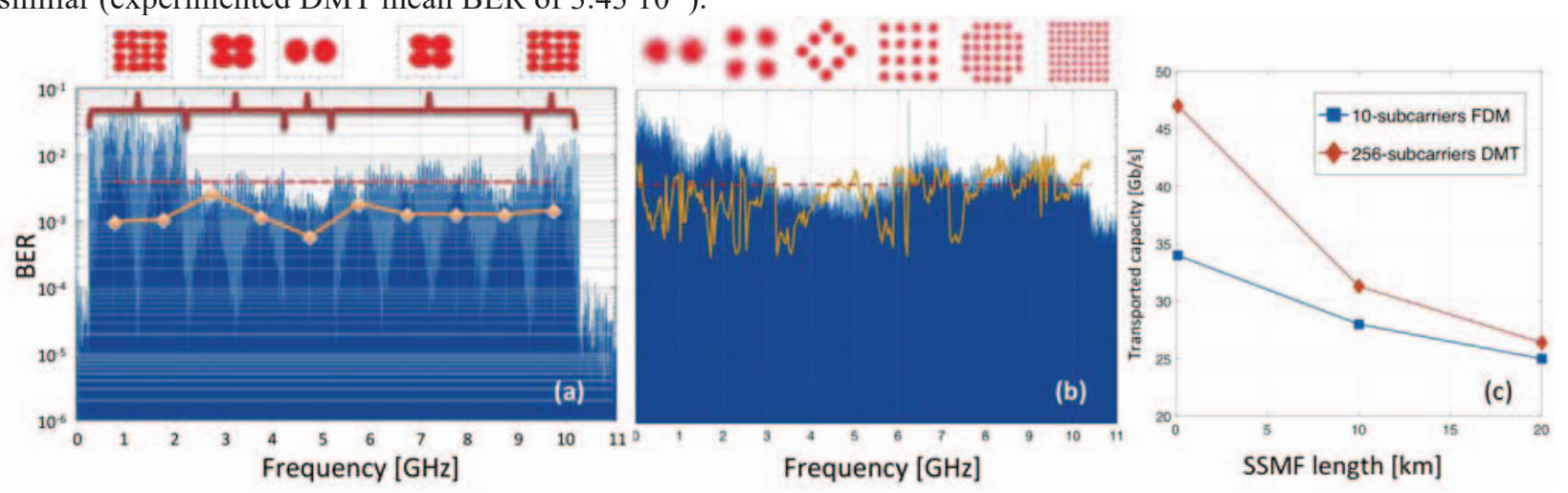

Fig. 1. Received electrical spectrum (blue) and subcarriers BER (orange) after 20-km SSMF in case of: a) 10-subcarriers FDM; b) 256-subcarriers DMT modulation (the BER threshold is in dashed red line). c) transported capacity vs. uncompensated SSMF reach.

\section{High capacity FDM upstream in bandwidth-limited RSOA-based PON}

For point-to-multipoint connections, as in PON architectures, FDM offers a high spectral efficiency with limited complexity. Contrary to OFDM, FDM does not require any synchronization for the subcarriers aggregation and it demands only a fraction of the sampling rate during the detection [4]. In FDM-based multiple access (FDMA) PON, the subcarriers can be advantageously reallocated according to the ONU subscriber type and requested services: by assigning one subcarrier or subsets of subcarriers to individual optical network units (ONUs), high-capacity US transmission can be supported thanks to the choice of the baud rate and the modulation format for each subcarrier.

In a PON architecture compliant with the NG-PON2 standard layout (Fig. 2 a), we demonstrate how to optimize the total US capacity with FDM in a colorless WDM reflective PON exploiting bandwidth-limited (4 GHz) reflective semiconductor optical amplifiers (RSOAs) [5]. In groups of 16 the ONU RSOAs are seeded by the same wavelength coming from the optical line terminal (OLT) and are directly modulated at 500 Mbaud each with 1 among 16 subcarriers. No inter-subcarrier guard-band is inserted with an initial 1-GHz gap. Fig. 2 b) shows the 43$\mathrm{km}$ SSMF propagation performance in terms of error vector magnitude (EVM) with 7\% overhead FEC BER threshold (experimented 31-dB optical distribution network losses). The central frequency subcarriers can be 16QAM modulated, achieving $2 \mathrm{~Gb} / \mathrm{s}$ data rate per ONU, while owing to the RSOA bandwidth limitation the higher frequency subcarriers require the more robust QPSK modulation (as for the lower frequency ones due to the initial band gap). $23 \mathrm{~Gb} / \mathrm{s}$ capacity per wavelength is achieved with a 9-GHz FDM signal spectrum, thus by means of 4 US WDM carriers an overall throughput of $92-\mathrm{Gb} / \mathrm{s}$ can be supported with about a tenfold increase in US capacity with respect to the NG-PON2 standard.

\section{Joint exploitation of FDM and MDM for advanced high-capacity applications}

The FDM subcarriers allocation and modulation can be also designed to face the channel crosstalk, for example introduced by non-ideal multiplexing. This is the case of MDM when, in order to achieve reduced-complexity implementation avoiding coherent detection and massive MIMO, weakly-coupled propagation is chosen combined to passive and all-optical multiplexers/demultiplexers (MUXs/DEMUXs), e.g. photonic lanterns (PLs) [6]. Despite the significant intermodal crosstalk, the system capacity can be increased thanks to joint exploitation of MDM and FDM, by effectively assigning the FDM subcarriers of the signals transported into the fiber by the optical modes, according to the intermodal crosstalk. 
(a)

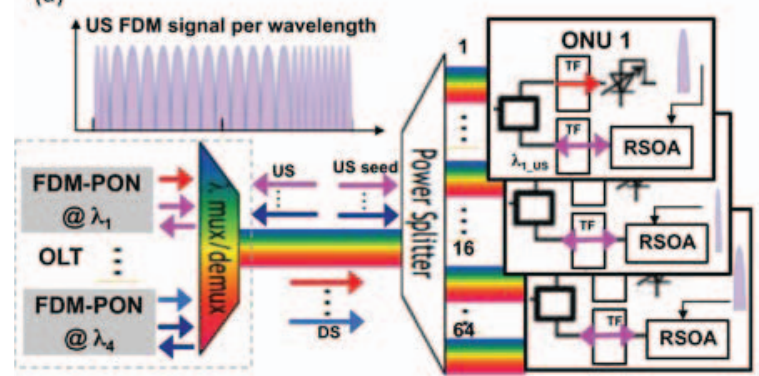

(b)

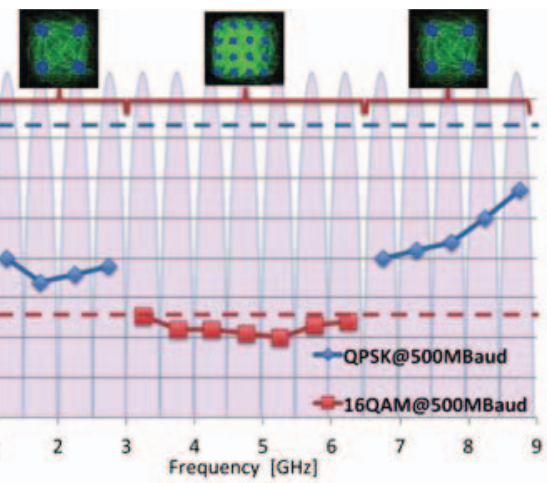

Fig. 2. a) RSOA-based FDM PON scheme for US transmission. b) Measured EVM for 16 FDM subcarriers modulated at 500 Mbaud after 43 -km SSMF. The QPSK and 16QAM EVM targets (dashed blue and red line, respectively) are correspondent to $3.8 \cdot 10^{-3} \mathrm{BER}$.

This strategy can be exploited in the mobile fronthaul (MFH) network of future radio access links, where a huge number of antenna elements will be connected. Although FDM mobile signals assisted by analog and digital signal processing have been already exploited [7], an effective combination of FDM and MDM offers a further degree of freedom in the design of wireless antennas assignment performed by beamforming, able to overcome the limitations of intermodal crosstalk. We demonstrate [8] that after $10-\mathrm{km}$ propagation in a few mode fiber, when employing commercial mode MUX/DEMUX, the joint exploitation of FDM and MDM (where the same number of antenna elements can be supported by different combinations of FDM subcarriers and modes) improves the MFH performance (as shown in Fig. 3 b) allowing to achieve capacities near the ideal ones.
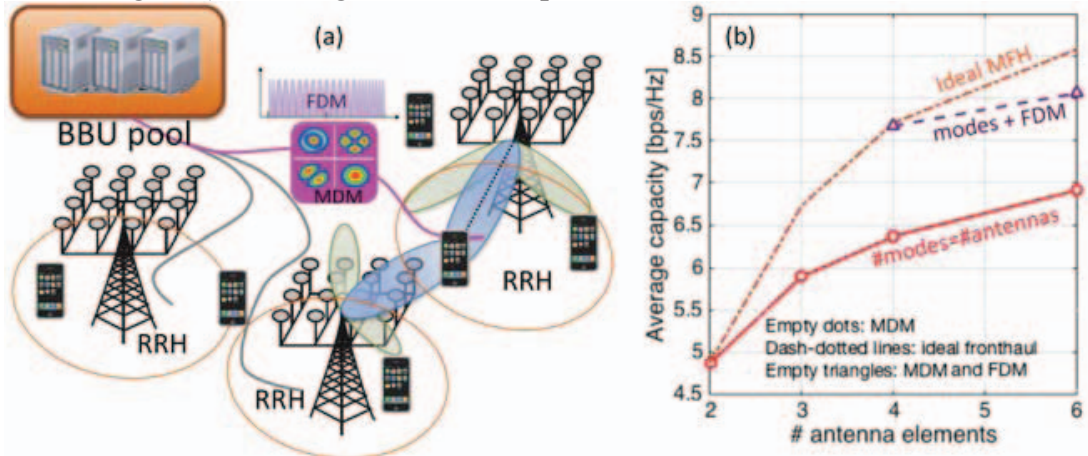

Fig. 3. a) Mobile fronthaul based on MDM and FDM. b) Average capacity per user as a function of number of antenna elements exploiting MDM with commercial mode MUX/DEMUX and FDM in case of 10-km SSMF for $30 \mathrm{~dB}$ radio SNR.

\section{Conclusions}

We have shown recent progress on the exploitation of FDM modulation to achieve high transported capacity in short-medium reach applications. More specifically, we have demonstrated the capabilities to face the bandwidth limitations of optical components, such as VCSELs and RSOAs, useful in the development of future low-cost and energy-efficient systems. FDM intrinsic flexibility is particularly suitable for point-to-multipoint applications, also in combination with weakly-coupled MDM, making FDM very attractive for next generation high-capacity PON and mobile fronthaul networks.

\section{Acknowledgements}

The authors thank L. Combi, P. Martelli, M. Martinelli, A. Matera and U. Spagnolini for the support to the research.

\section{References}

[1] M. Sezer Erkilic et al., "Performance comparison of single-sideband direct detection Nyquist-subcarrier modulation and OFDM," J. Lightwave Technol. 33, 10, 2038-2046 (2015).

[2] M. Ortsiefer, W. Hofmann, J. Rosskopf and M.-C. Amann, VCSELs - Springer Series in Optical Sciences 166 (Springer, 2013 ), Chap. 10.

[3] A. Gatto et al. "Very high-capacity short-reach VCSEL systems exploiting multicarrier intensity modulation and direct detection," Opt Express 24, 12769-12775 (2016).

[4] S. Straullu et al., "Optimization of reflective FDMA-PON architecture to achieve $32 \mathrm{~Gb} / \mathrm{s}$ per upstream wavelength over $31 \mathrm{~dB}$ ODN loss," J Lightwave Technol. 33, 2, 474-480 (2015).

[5] A. Gatto et al., "RSOA-based FDM PON upstream with flexible multiple access capabilities in a NG-PON2 compliant architecture," IEEE J. Opt. Commun. Netw. 8, 302-307 (2016).

[6] D. Yu, S. Fu, Z. Cao, et al.,"Mode-dependent characterization of photonic lanterns," Opt. Lett. 41,2302-2305 (2016).

[7] X. Liu et al., "Efficient mobile fronthaul via DSP-based channel aggregation," J. Lightwave Tech. 34, 1556-1564 (2016).

[8] L. Combi et al., "Radio-over-Modes for C-RAN architecture with smart optical resources assignment," submitted to IEEE ICC 2017. 